\title{
Guidelines for red blood cell and plasma transfusion for adults and children
}

\begin{abstract}
Editorial Note. The Guidelines which follow were developed in Canada by an Expert Working Group of the Canadian Medical Association, and issued in June 1997, but they are of global validity. The Group was chaired by Dr Edward Crosby of Ottawa. The Guidelines represent a remarkably complete and concrete overview of the principles which should be respected if transfusions are to be used in appropriate situations and to achieve their goal without undue risk to the patient. They are complemented by a "Patients' Guide", an extensive list of references and a series of background papers which are available on request from Dr Anne Carter, Director of Health Programs, CMA, 1867 Alta Vista Drive, Ottawa ON K1G $3 \mathrm{Y} 6$.
\end{abstract}

Ideally, practice guidelines are intended to assist practitioners and patients to make decisions about appropriate health care. They are not intended to provide a rigid prescription for care. Guidelines should be discussed, adopted or modified according to local clinical needs and constraints.

\section{Why do we need Canadian guidelines for red blood cell and plasma transfusion?}

Many available guidelines are outdated, because the science and the blood system have changed dramatically in the last decade. Some guidelines reflect values that are not relevant to Canada; others rely heavily on "expert" opinions rather than critical review of evidence. Although in some areas of transfusion inadequate scientific evidence exists and "expert" opinions are necessary, in other areas sufficient evidence exists and should be used to generate recommendations. As well, some guidelines deal only with surgical populations; for other populations, patients with chronic anemia and, particularly, children, guidelines do not exist.

The great many publications on transfusion medicine in the last decade require synthesis and interpretation. New blood programs, such as autologous predeposit and intraoperative cell salvage, lack criteria for best integrating them into the predominantly allogeneic system. Critical evaluation of current transfusion practices in Canada may lead to new perspectives in clinical decision-making and applying new information and new choices.

Those generating guidelines have certain values that influence how the evidence is reviewed and the guidelines are elaborated. Two principles guided the Expert Working Group (EWG): the guidelines should permit optimal patient care while fostering prudent clinical use of the precious allogeneic blood supply. The EWG considered avoidance of transfusion to be ideal to the extent that avoidance was not likely to be a more serious risk than the transfusion. In addition, the members believe that, whenever possible, patients should be involved in transfusion decisions. 


\section{Informed consent and information disclosure}

The EWG had some difficulty reaching consensus on some issues related to obtaining informed consent for red blood cell and plasma transfusion. Members agreed that health care workers should inform patients about the possibility and likelihood of blood transfusion, and solicit and answer any questions the patient might have. Beyond that point, members had difficulty providing guidelines for informed consent that reconcile requirements for full disclosure irrespective of the clinical scenario with a more pragmatic approach that considers disclosing transfusion-related information in light of the patient's clinical situation.

The EWG believes that the risk associated with red blood cell and plasma transfusion must be placed in the context of the overall risk associated with an illness or procedure. Blood transfusion is often given in situations, such as surgery, chemotherapy or critical illness, where the risk from transfusion is small compared with the overall risks. Undue emphasis on the risks from blood transfusion was thought by some to be both unreasonable and imprudent, as it could confuse patients about the absolute and relative risks they are facing. For example, coronary artery bypass grafting has an approximate composite risk of stroke, myocardial infarction and death of $1 \%$ to $2 \%$. Is it then necessary or even appropriate to do more than advise patients that they have a $10 \%$ to $40 \%$ probability of receiving a blood transfusion, with which there are associated remote but real risks, then solicit the patient's response and questions.

The EWG agreed that, where clinically appropriate, patients should be advised that alternatives to allogeneic transfusion exist, but members could not reach consensus on the need to discuss them in detail, given their inconsistent availability and varying effectiveness in averting allogeneic exposure and preventing transmissible diseases.

Finally, the EWG agreed that patients should be informed that they have received red blood cells or plasma as soon as possible following transfusion, but could not reach consensus on who should inform them (e.g., treating physician, prescribing physician, blood bank, hospital administration) or how to ensure that they were informed.

\section{Transfusion threshold}

These guidelines differ from others in not explicitly recommending a threshold hemoglobin concentration or range of concentrations at which patients should be transfused. EWG members agreed that there was no evidence to support the generation of such thresholds. The EWG believes that the decision to transfuse should be made after review of the patient and the clinical situation; in addition, timely measurement of hemoglobin concentrations should be considered.

\section{Volumes of plasma to be administered}

These guidelines differ from others in not explicitly stating volumes of plasma to be administered. The EWG agreed that published practical guides for appropriate initial-dose volumes in given clinical situations are prudent. It was recommended that plasma be given in doses calculated to achieve a minimum of $30 \%$ of normal concentrations for most plasma factors (usually achieved with administration of $10-15 \mathrm{~mL}$ of plasma per kilogram body weight), except for urgent reversal of warfarin anticoagulation, for which $5-8 \mathrm{~mL} / \mathrm{kg}$ will usually suffice. 
However, these values are derived not from systematic assessments of therapy, but from synthesis of physiologic measurements of factor concentrations, hemostatic function and clinical observations of the effect of plasma administration on abnormal coagulation. Ongoing clinical and laboratory assessments are necessary to determine subsequent action.

\section{Information about infectious risks of transfusion}

In the past, physicians prescribing blood products have not had accurate and readily available information on the infectious risks of blood transfusion. No single national agency has assumed or been assigned to provide physicians or the community at large with this information. The EWG believes that a single agency should have the responsibility to provide this information, but could not reach a consensus about which agency should be delegated or whether the EWG had the mandate to make such a proclamation. However, the EWG believes that those who constitute the Canadian blood system have the responsibility to do this without delay.

\section{Methods}

These guidelines were developed according to the CMA's Guidelines for Canadian Clinical Practice Guidelines. They cover red blood cell and plasma transfusions in adults and in children over 4 months of age. For infants under 4 months, the Canadian Paediatric Society has developed guidelines for transfusion of red blood cells. Informed consent and the infectious risks related to the transfusion of these components are also addressed.

Broad guidelines and principles have been developed; specific clinical conditions or indications for specialized blood components, such as leuko-reduced or irradiated components or components at low risk of transmitting cytomegalovirus, are not addressed. The specific development process comprised 4 steps:

\subsection{Background research}

In this initial phase, consultants with the required expertise were commissioned to provide:

- a review of the scientific evidence supporting clinical indications for transfusion. For all sections, excluding that relating to autologous transfusion, a search of MEDLINE from January 1966 to July 1996 for all languages was constructed using the following medical subject headings: blood transfusion, erythrocyte transfusion and blood component transfusion. Manual searches of bibliographies of relevant reviews, guidelines and textbooks were also undertaken. For autologous transfusion, a recent meta-analysis was used as the evidence base;

- a review of relevant ethical and legal issues;

- an evaluation of physicians' current needs related to transfusion and counselling practices as expressed in 9 focus groups convened in Toronto, Vancouver, Ottawa, Montreal and Cornwall.

\subsection{Guideline development}

- Expert Working Group: Organizations representing various stakeholders were asked to suggest members for the EWG. All sponsors reviewed the curricula vitae of potential members. By this process, 8 candidates were selected and appointed to the EWG by the CMA's Board of Directors. 
- Advisory panel: A larger group was convened to reflect the concerns of patients and practising providers and to provide feedback to the EWG. The 26 members of the Advisory Panel were appointed by their organizations, to represent their stakeholder group on the panel.

- Levels of evidence: The definition of the levels of evidence used to grade the recommendations in these guidelines is a modified version of that used by the Canadian Task Force on the Periodic Health Examination:

- Level I: Evidence obtained from at least one properly randomized controlled trial.

- Level II: Evidence obtained from well-designed controlled trials without randomization, cohort or case-control analytic studies, preferably from more than one centre, or research or evidence obtained from comparisons between times or places with or without the intervention.

- Level III: Opinions of respected authorities, based on clinical experience, descriptive studies or reports of expert committees.

- Not applicable (N/A): opinions of the EWG about issues that cannot be evaluated using accepted study designs.

- Consensus: The EWG reviewed both the background research identified above and additional articles retrieved by its members. The evidence was synthesized and draft positions were written, reviewed and revised during both face-to-face and teleconference meetings. A composite document was then constructed and presented at an assembly of the EWG and Advisory Panel. This document was further revised by the EWG, then circulated to the Advisory Panel, the participating organizations and the consultants for written commentary. This commentary was reviewed by the EWG in a series of teleconferences and the final document was prepared and submitted to the Canadian Medical Association Journal for peer review.

\subsection{External review}

Members of the EWG and the Advisory Panel suggested external reviewers who were members of medical organizations and other health professional associations, experts in the field and other interested parties. The comments of the external reviewers were considered by the EWG for incorporation into the final draft of the guidelines.

\subsection{Endorsement}

All organizations who appointed representatives to the Advisory Panel were contacted to obtain an official endorsement of the final draft of the guidelines.

\section{Issues related to informed consent}

The following is a general discussion of issues related to informed consent. For a more complete discussion, physicians are referred to Consent: A Guide for Canadian Physicians.

\subsection{What is informed consent?}

Informed consent is undertaken by a patient and a physician to make a therapeutic decision; it allows the patient to preserve his or her primary role in making the decision to undergo a treatment 
or procedure. As part of this process the physician must conform to a standard of disclosure about the risks and benefits of a particular therapy or procedure: to disclose information that the physician knows or ought to know and a reasonable person in the patient's position would wish to know before making the decision.

When possible, discussions about transfusion should take place early enough to allow time to procure feasible alternatives such as autologous transfusion. The language should be easily understood by the patient; a preprinted patient information sheet may be helpful but cannot supplant discussion. The discussion must include: description of the blood product or component to be transfused; the expected benefits, including possible consequences if transfusion is not given; the associated risks, including life-threatening risks; and appropriate alternatives. The patient must be given an opportunity to ask questions and the physician must ensure that the patient understands the explanation. Confirmation that the consent discussion has taken place is best noted in the patient's (hospital) chart. Signing a separate consent form for blood transfusion is of no particular advantage and cannot replace the discussion that must occur.

Transfusion of blood products raises myriad ethical issues including: access to resources; access to alternative treatments; social, psychological, physical and emotional well-being of the patient; differing perceptions of risk; and potential conflicts of opinion and belief between the physician and the patient. The physician should be aware of these issues and the diverse cultural differences and beliefs in our society and act accordingly. A blood transfusion is often only part of a larger medical or surgical intervention, and the risks of the transfusion may be small compared with the total risk associated with the intervention. During the consent discussion, it may be appropriate that the emphasis placed on the various risks (i.e., that of the blood transfusion) reflect their relative magnitude in the whole process.

\subsection{Special considerations: emergencies, children and incompetent adults}

\subsubsection{Emergencies}

Physicians may treat a patient without consent when the treatment is necessary to preserve a patient's life or health and

- it is not possible to obtain consent because the patient is not mentally or physically capable of giving consent (either inherently or due to his or her medical condition);

- no substitute decision-maker is available;

- there is no evidence of advance directives.

This also applies to emergencies involving children if their parents or guardians cannot be reached. If consent to treatment is refused, no treatment may be given, but the physician should ensure that the patient is competent to refuse treatment.

\subsubsection{Children and adolescents}

The responsibility for making decisions on behalf of a minor normally rests with the parents or legal guardians. In the case of divorced parents, decision-making power rests with the parent who has legal custody. Decisions should be based on the best interests or welfare of the child rather than those of the parents. Canadian courts have not permitted parents to refuse life-saving transfusions (or any other life-saving treatment) for their children on religious grounds. If a physician believes that refusal of a transfusion might endanger the child's life or health, and discussions with the parents or guardians 
fail to alter the decision, the physician is generally obliged, under provincial child protection laws, to notify and seek assistance from the appropriate authorities.

Generally, minors may consent to or refuse medical treatment if they are competent and capable of appreciating the full nature and consequences of the treatment or refusal of treatment. Although determination of capacity of a minor is the responsibility of the physician, the physician must be aware that provincial legislation may establish presumptive ages of capacity and specific conditions under which parents, guardians or child protection agencies must be notified. In most provinces, child protection legislation requires that physicians notify authorities if, for example, they feel a child's life, health or safety is in danger. Some statutes make such reports mandatory for minors who are under a certain age even if the minor would otherwise be sufficiently mature to consent to or refuse treatment.

\subsubsection{Incompetent adults}

For adults who have never been competent, decisions must be in the patient's best interest. For adults who were once competent but have become temporarily or permanently incompetent, their prior wishes regarding treatment, to the extent that these are known or can be determined, should be respected. Some jurisdictions have passed legislation that provides for substitute consent and prior treatment directions (advance directives) and physicians should be aware of the provisions within their own jurisdiction. The provincial medical colleges and the Canadian Medical Protective Association are useful resources in these matters.

\subsubsection{Informing transfusion recipients}

Whenever possible, patients (or their guardians) should be informed that they have received a red blood cell or plasma transfusion. Providing this information respects the patient's autonomy and encourages more individual responsibility for health care.

\subsection{Refusal of transfusion}

A competent adult is entitled to refuse or cease any treatment for any reason. Refusal occurs most often among Jehovah's Witnesses, whose religion precludes receipt of blood products. The refusal is valid under any circumstances, even when it will result in the patient's death. Note that this does not apply to children (see Children and adolescents, above). The physician should ensure and, if possible, record that the patient's refusal is fully informed and that the patient is aware of the consequences of his or her decision. Exploration of issues such as depression, which may affect the patient's capacity and choice, should be undertaken. Although the physician who properly advises the patient is not liable for the consequences of a refusal, physicians are advised to seek second opinions and legal advice if the refusal is likely to lead to serious morbidity or death.

\subsection{Recommendations regarding informed consent}

1. Patients should be informed that transfusion of red blood cells, plasma or both is a possible element of the planned medical or surgical intervention and be provided with information about the risks, benefits and available alternatives.

Level of evidence: N/A

2. When feasible, the patient's consent to a transfusion of red blood cells, plasma or both should be obtained and recorded in the patient's medical chart. 
3. The physician overseeing the care of the patient should be responsible for obtaining informed consent for red blood cell or plasma administration.

Level of evidence: N/A

4. Patients should be informed that they have received a red blood cell or plasma transfusion subsequent to its administration.

Level of evidence: N/A

\section{Allogeneic red blood cell transfusion}

The standard unit of whole blood collected consists of approximately $450 \mathrm{~mL}$ of blood taken into $63 \mathrm{~mL}$ of anticoagulant. Red blood cells are prepared by removing supernatant plasma from a whole blood donation after centrifugation. The characteristics of this component vary depending on the anticoagulant-preservative solution used (Table 1). In the average adult, each unit of blood should raise hemoglobin by $10-15 \mathrm{~g} / \mathrm{L}$.

\subsection{Oxygen transport and the physiologic responses to anemia}

The simplest method of reducing the frequency of allogeneic blood transfusion is to withhold transfusion until more severe levels of anemia are reached. However, the optimum threshold for the initiation of transfusion therapy is not yet defined and, clearly, the level of anemia permitted cannot be such that tissue oxygen delivery $\left(\mathrm{DO}_{2}\right)$ or consumption are compromised. The oxygen carrying capacity of blood is measured either indirectly by measurement of the red blood cell concentration (the hematocrit) or directly by determining hemoglobin concentration ([Hb]). Although both measures are employed in research reports, $[\mathrm{Hb}]$ is more commonly used in the context of clinical medicine.

$\mathrm{DO}_{2}$ is the product of tissue blood flow and arterial oxygen content; at the whole body level these factors are represented by the product of cardiac output $(\mathrm{CO})$ and the arterial oxygen content $\left(\mathrm{CaO}_{2}\right)$.

$$
\mathrm{DO}_{2}=\mathrm{CO} \times \mathrm{CaO}_{2}
$$

$\mathrm{CaO}_{2}$ depends on $[\mathrm{Hb}]$ and the percentage of $\mathrm{Hb}$ saturated with oxygen. $\mathrm{CO}$ is affected by both preload (venous return) and afterload. Blood flow is determined by resistance to flow in the vascular

Table 1

Characteristics of red blood cell components depending on anticoagulant used during collection

\begin{tabular}{lll}
\hline & \multicolumn{2}{c}{ Anticoagulant-preservative solution } \\
\cline { 2 - 3 } Characteristic & \multicolumn{1}{c}{ CPDA-1 } & \multicolumn{1}{c}{ CP2D } \\
\hline Additive (after plasma removed) & 0 & $100 \mathrm{~mL}$ AS-3 (Nutricel) \\
Hematocrit & $0.72-0.79$ & $0.45-0.65$ \\
Red blood cell volume & Approx. $200 \mathrm{~mL}$ & Approx. $200 \mathrm{~mL}$ \\
Plasma volume & Approx. $90 \mathrm{~mL}$ & Approx. $60 \mathrm{~mL}$ \\
Shelf life & 35 days & 35 days* \\
\hline
\end{tabular}

Note: $\mathrm{CPA}-1$ = citrate phosphate dextrose adenine; $\mathrm{CP} 2 \mathrm{D}=$ citrate phosphate double dextrose. ${ }^{*}$ Currently licensed by the Bureau of Biologics for 35 days but has the potential for 42 days shelf life. The Canadian Red Cross Society is requesting that the shelf life be extended to 42 days. 
bed and the perfusion pressure driving flow through the bed. Blood is more viscous (inherently resistant to flow) at lower flow rates. Thus, viscosity is highest in venules and lowest in the aorta. Viscosity, independent of flow rate, is primarily a function of red blood cell concentration. Reduction in the red blood cell concentration lowers blood viscosity and reduces the resistance to flow. With progressive reduction there is an incremental rise in $\mathrm{CO}$. There is some inconsistency in reports as to the $[\mathrm{Hb}]$ at which $\mathrm{CO}$ begins to rise. In adults, the higher $\mathrm{CO}$ is initially a result of enhanced preload and a decreased afterload. The augmented venous return is a result of the profound reduction in viscosity and a passive increase in blood flow in the postcapillary venules. The decrease in afterload is produced by the reduction in the viscosity component of the systemic vascular resistance. In children, the increase in $\mathrm{CO}$ is more dependent on increased heart rate and less on enhanced stroke volume than in adults.

Blood with a low $[\mathrm{Hb}]$ has decreased oxygen carrying capacity. To compensate for this and to maintain oxygen delivery, tissues may augment blood flow, either by recruiting capillaries or by increasing flow through the existing capillary network. Tissues may also increase oxygen extraction ratios (ER). Enhanced oxygen extraction may occur particularly in tissue beds that normally consume a small proportion of the available oxygen. In supply-dependent beds, such as in the heart, higher ERs occur under normal conditions. In these beds, to preserve oxygen consumption and aerobic metabolism, regional blood flow must increase proportionally more than the increment in $\mathrm{CO}$. As $[\mathrm{Hb}]$ decreases to $50 \mathrm{~g} / \mathrm{L}$ (hematocrit 0.15 ), there is evidence of decreased myocardial oxygen consumption, which may be due to impairment in myocardial oxygen extraction. In animals, the onset of coronary lactate production (anaerobic metabolism) occurs at a $[\mathrm{Hb}]$ below $35 \mathrm{~g} / \mathrm{L}$ (hematocrit 0.10 ). In a model of coronary stenosis, this anaerobic state occurs at a $[\mathrm{Hb}]$ of $60-70 \mathrm{~g} / \mathrm{L}$. These values also coincide with the onset of ventricular wall motion abnormalities.

Tissue oxygenation may be improved with a shift to the right of the Hb-oxygen dissociation curve (to a higher $\mathrm{P}_{50}$ ). This can be achieved through increased red blood cell levels of 2,3-diphosphoglycerate (2,3-DPG) and decreased $\mathrm{pH}$, both of which facilitate oxygen unloading in the tissues. Changes in the $\mathrm{Hb}$-oxygen dissociation curve resulting from changes in red blood cell 2,3-DPG levels take 12-36 hrs to occur and increase with decreasing $[\mathrm{Hb}]$. Because of these adaptations, in chronic anemia a $50 \%$ decrease in oxygen carrying capacity is accompanied by only a $25 \%$ decrease in oxygen availability.

The physiologic compensations for a decreased [Hb] must be sufficient to balance the lower oxygen carrying capacity and maintain tissue oxygen delivery. The optimum [Hb] is the level that allows for the greatest oxygen delivery at the lowest energy cost to the organism. In terms of whole-body oxygen delivery, Messmer concluded that the optimum [Hb] was $100 \mathrm{~g} / \mathrm{L}$ (hematocrit 0.30 ). As [Hb] was decreased from $150 \mathrm{~g} / \mathrm{L}$ to $100 \mathrm{~g} / \mathrm{L}$, while maintaining normal circulating blood volume, reduction in viscosity was sufficient to allow increased blood flow such that systemic oxygen transport capacity increased. With further reduction in $[\mathrm{Hb}]$, oxygen delivery declined so that, at $[\mathrm{Hb}]$ about $90 \mathrm{~g} / \mathrm{L}$, tissue oxygen delivery was at or below preanemic levels. Because oxygen delivery remained relatively constant between $[\mathrm{Hb}] 90 \mathrm{~g} / \mathrm{L}$ and $150 \mathrm{~g} / \mathrm{L}$, there appeared to be little rationale for transfusing red blood cells to patients with [Hb] already in this range to increase oxygen delivery. Messmer's conclusion that systemic oxygen transport was optimum at [Hb] of $100 \mathrm{~g} / \mathrm{L}$ was supported by whole-body physiologic measurements and cannot be extrapolated to support conclusions regarding regional circulations. Furthermore, although Messmer's assertions are widely acknowledged and help form the basis for current transfusion strategies, they disagree with the work of other investigators. Le Merre and co-workers reported that systemic oxygen transport capacity decreased with even moderate hemodilution and argued that increased tissue extraction of oxygen was the major compensating factor. 
Athough the mechanisms for compensation remain to be clarified, clinical experience supports Messmer's conclusions; that is, there seems to be little clinical gain in transfusing most patients whose $[\mathrm{Hb}]$ is in the range $90-150 \mathrm{~g} / \mathrm{L}$. The lack of measured benefit of transfusion is supported by the work of Hébert and colleagues, who in a multicentred, randomized, controlled clinical trial, evaluated the effects of a restrictive transfusion threshold ([Hb] 70-90 g/L) compared with a more liberal one $(100-120 \mathrm{~g} / \mathrm{L})$ in critically ill patients developing a $[\mathrm{Hb}]$ of less than $90 \mathrm{~g} / \mathrm{L}$ in the first $72 \mathrm{hrs}$ after admission to an intensive care unit (ICU). The study groups were demographically similar. The number of units of blood administered was significantly smaller for the patients with the restrictive threshold; yet there was no difference noted in ICU 30-day and 120-day mortality. Although the findings are encouraging, this was a small study and confirmation following evaluation of larger groups is required.

Messmer's work provides a basis for estimating the optimum [Hb], but the experience of Jehovah's Witness patients provides data on the lowest tolerable concentration, perhaps a more appropriate marker to justify transfusion. Viele and Weiskopf reviewed 54 publications involving 134 patients with moderate to severe anemia. The overall case fatality rate was $37 \%$, and all patients whose deaths were attributed to anemia died with [Hb] less than $50 \mathrm{~g} / \mathrm{L}$. Considering only anemia-related deaths, the case fatality rate was $27 \%$ for patients who were under 50 years of age and $53 \%$ for patients over 50 years. No patients with a [Hb] of 50-80 g/L died because of their anemia. Although these findings may reassure clinicians that very low $[\mathrm{Hb}]$ is tolerated in many younger patients and some older ones, there is a selection bias in these reports. Obviously, severely anemic survivors are more frequently reported than nonsurvivors, and the risk of severe anemia may thus be understated. Furthermore, mortality is not the only endpoint to be avoided with blood transfusion; these papers provide little information about morbidity-avoidance with transfusion.

Although there is little direct clinical evidence, much of the risk posed to the patient by a low $[\mathrm{Hb}]$ probably relates to the ability of the heart to tolerate anemia. Carson and co-workers reported a retrospective cohort study of 1958 adults (over 18 yrs of age) who underwent surgery and refused blood transfusion between 1981 and 1994. There was an increase in the risk of death associated with a lower preoperative [Hb], and this risk was enhanced by the presence of cardiovascular disease. Mortality within 30 days was $1.3 \%$ in patients with $[\mathrm{Hb}]$ of $120 \mathrm{~g} / \mathrm{L}$ and increased in a roughly linear fashion to $33.3 \%$ in patients with $[\mathrm{Hb}]$ less than $60 \mathrm{~g} / \mathrm{L}$. In addition, the effect of blood loss on mortality was greater in patients with a lower preoperative $[\mathrm{Hb}]$. The authors concluded that a low preoperative $[\mathrm{Hb}]$ or substantial blood loss during surgery increases the risk of death or perioperative morbidity and that this effect is larger in patients with cardiovascular disease.

Many physiologic mechanisms compensate for acute anemia and preserve tissue oxygen delivery. However, if these compensatory mechanisms are not intact, oxygen delivery may not be preserved and organ dysfunction may result. Older patients are less tolerant of low [Hb] levels; they are less able to increase heart rate and stroke volume (and hence $\mathrm{CO}$ ) in response to exercise. In elderly patients with low $[\mathrm{Hb}]$ but normal blood volume, oxygen delivery is decreased as a result of failure to increase $\mathrm{CO}$. Oxygen consumption is maintained by increased extraction. This finding has been reported in healthy patients, patients with medical disease excluding patients with heart disease and patients with recognized coronary artery disease. Although chronologic age does not necessarily parallel physiologic age, and there are wide individual differences in functional capability in a population, the compensatory mechanisms that preserve oxygen delivery generally are reduced in the elderly.

The perception that the elderly are at greater risk than younger cohorts if they become anemic presumably is related, not only to their reduced physiologic compensatory mechanisms, but also to 
the higher incidence of coronary artery disease and the resultant reduced capacity to increase coronary artery blood fow and, perhaps, CO. This may limit the degree of hemodilution that will be tolerated by these patients because increased flow is an essential compensatory mechanism. Relatively modest hemodilution plus depleted or exhausted coronary vasodilator reserve may compromise ventricular metabolism and function. Electrocardiographic evidence of ischemia was observed in $22 \%$ of patients with pre-existing impaired left ventricular function who were hemodiluted, but was not seen in patients with normal left ventricular function. This report suggests that, in the presence of moderate coronary artery stenosis, flow may not increase sufficiently to offset the loss of oxygen carrying capacity caused by hemodilution, and ischemic cardiac dysfunction may result from even a modest reduction in $[\mathrm{Hb}]$. Patients with critical vessel stenosis or pre-existing left ventricular dysfunction may not tolerate hemodilution to any degree. An association has been reported between low perioperative $[\mathrm{Hb}]$ levels and the occurrence of myocardial ischemia and infarction. Finally, Carson and co-workers concluded that patients with cardiovascular disease had a much greater risk of preoperative death than patients without cardiovascular disease when their preoperative $[\mathrm{Hb}]$ was $100 \mathrm{~g} / \mathrm{L}$ or less. Coronary artery disease likely constitutes an important factor in determining a patient's tolerance of low [Hb].

Much of the information on tolerable levels of anemia comes from research in which anemia is the predominant physiologic stressor. In clinical medicine, the concurrent presence of underlying disease states, both acute and chronic, metabolic derangements or disturbances in oxygen transport may alter the patient's tolerance of anemia. However, there is insufficient explicit information to justify conclusions and meaningful comment in these specific situations.

For clinically stable patients who are not at risk for coronary artery disease, transfusion is more likely to be beneficial when $[\mathrm{Hb}]$ is less than $60 \mathrm{~g} / \mathrm{L}$, but not when $[\mathrm{Hb}]$ is greater than $80 \mathrm{~g} / \mathrm{L}$, as long as normal blood volume is maintained and patient assessment is ongoing. Critically ill patients and those at risk for coronary artery disease are less likely to be as tolerant of low [Hb] and will likely benefit from maintenance of a higher range of $[\mathrm{Hb}]$ than patients not at risk.

Indices of oxygen delivery and tissue oxygenation may accurately indicate the need to transfuse red blood cells; however, invasive monitoring is required to generate these indices. Thus, decisions about transfusion must often be made on the basis of available but less informative data, including patient characteristics (i.e., age, presence of heart disease), the clinical context (i.e., stable with no ongoing bleeding versus ongoing blood loss), measured vital signs and the patient's tolerance of the situation. In some situations, particularly in the perioperative period and also in critically ill, sedated and ventilated patients, medications may further mask the expected hemodynamic indices, and patient characteristics and the clinical context may have a greater role in the decision to transfuse red blood cells.

In some situations, transfusion of multiple units is appropriate - typically during resuscitation from a major hemorrhage. However, the routine transfusion of multiple units in less urgent situations appears to be common and should be re-evaluated. Instead, physicians should consider transfusing one unit at a time with assessment after each unit to avoid unnecessary exposure.

\subsection{Principles specific to acute blood loss}

When blood loss is acute, initial reductions in total arterial oxygen content are usually well tolerated because of compensatory increases in CO. Although determined by the rate of blood loss, the lower limit of human tolerance to acute anemia has not been established, but oxygen delivery will probably be adequate in most people with a $[\mathrm{Hb}]$ above $70 \mathrm{~g} / \mathrm{L}$, assuming that oxygen delivery is not compromised 
by other physiologic disturbances. However this is not always a valid assumption. The effects of medications, pre-existing disease and hypothermia have been described well and must be considered when assessing an individual patient's response to acute blood loss. Barring such other factors, tissue oxygenation appears to be maintained and anemia well tolerated at a hematocrit as low as $0.18-0.25$ $([\mathrm{Hb}]$ of $60-80 \mathrm{~g} / \mathrm{L})$.

Measured $[\mathrm{Hb}]$ will frequently be misleading during acute blood loss because it depends on the rapidity and degree of blood loss and the effects of fluid resuscitation (acute hemodilution). With ongoing blood loss, by the time a $[\mathrm{Hb}]$ is reported (from a sample drawn earlier), the actual oxygen carrying capacity may have changed.

There is currently no evidence to support a threshold value for initiating blood transfusion in the case of acute blood loss. All recent reviews have refuted the concept of a transfusion threshold, most concluding that insufficient evidence exists to support a single hemoglobin requirement. The decision to administer red blood cell transfusions should be determined by evaluating the rate of ongoing blood loss, evidence of end-organ compromise and the risk or presence of coronary artery disease.

Acute blood loss may occur in a variety of clinical situations. The most important clinical features are the amount of blood loss and the likelihood of continuing losses. The primary treatment goals in this setting are to restore intravascular volume, to ensure sufficient oxygen carrying capacity and to stop the blood loss as soon as possible. Management of a patient with acute blood loss should also include other measures to maximize the oxygenation of circulating blood (such as airway management, administration of oxygen and treatment of pulmonary injuries). The principles of management of hypovolemic shock are reviewed in the manual for the American College of Surgeons' advanced trauma life support course.

In cases of acute hemorrhage related to elective surgery, the availability of predeposited autologous blood units and measures to decrease blood loss (intraoperative and postoperative blood salvage, acute normovolemic hemodilution) may reduce the requirement for allogeneic blood cell transfusion.

\subsection{Principles specific to chronic anemia}

In determining the need for red blood cell transfusion, it is important to consider the differences between acute and chronic anemia. Acute anemia is often accompanied by hypovolemia which, at least initially, is often the major physiologic problem. The patient with chronic anemia is normovolemic or even hypervolemic. Also, in the case of chronic anemia, decisions usually need not be made rapidly; there is time to consider and discuss the role of transfusion therapy, and its benefits and risks with the patient. It is an ideal situation for involving a competent patient in the treatment plan.

Evaluating the symptoms of chronic anemia is not a simple process. In a study of adult patients with iron-deficiency anemia and $[\mathrm{Hb}]$ of $80-120 \mathrm{~g} / \mathrm{L}$, there was no correlation between the degree of anemia and the intensity of any of the symptoms of fatigue, irritability, palpitations, dizziness, breathlessness and headache. Furthermore, treatment with iron in amounts sufficient to increase [Hb] an average of $23 \mathrm{~g} / \mathrm{L}$ resulted in no greater improvement in any of the symptoms than did treatment with a placebo.

The relationship between the degree of anemia and functional status in patients with chronic renal failure was studied by the Canadian Erythropoietin Study Group. In a randomized, placebo-controlled study of 118 dialysis patients, investigators examined the effect on quality of life and exercise capacity of 3 levels of anemia: mean [Hb] of $70 \mathrm{~g} / \mathrm{L}$ (placebo group); mean [Hb] of $100 \mathrm{~g} / \mathrm{L}$ (low-dose erythropoietin group); and mean $[\mathrm{Hb}]$ of $120 \mathrm{~g} / \mathrm{L}$ (high-dose erythropoietin group). Quality of life, in global 
terms, was similar in all 3 groups. However, treated patients experienced significant improvement in symptoms (fatigue, depression, physical symptoms), although no difference was noted between the low- and high-dose treatment groups.

In general, otherwise healthy people display few symptoms or signs of anemia at rest when [Hb] is greater than $70-80 \mathrm{~g} / \mathrm{L}$, although they often show dyspnea with exertion; at $60 \mathrm{~g} / \mathrm{L}$ most patients will complain of some weakness; at $30 \mathrm{~g} / \mathrm{L}$ patients will complain of dyspnea at rest; and at $[\mathrm{Hb}]$ of $20-25 \mathrm{~g} / \mathrm{L}$ congestive heart failure frequently occurs. Children are amazingly tolerant of chronic anemia and may remain asymptomatic even when $[\mathrm{Hb}]$ is less than $50 \mathrm{~g} / \mathrm{L}$.

Before considering transfusion of red blood cells for the treatment of chronic anemia, it is essential to determine the cause of the anemia so that, where appropriate, treatment other than red blood cell transfusion may be used. Classic examples of anemias that may be severe but correctable by alternative therapies are iron-deficiency anemia in childhood and pernicious anemia in adults.

If red blood cell transfusion is considered necessary for the immediate treatment of a chronic anemia, the goal need not be to attain a normal $[\mathrm{Hb}]$ but rather to attain a $[\mathrm{Hb}]$ that will avert the danger of inadequate tissue oxygenation or cardiac failure. When red blood cell transfusion is considered for the long-term treatment of chronic anemia; treatment goals (other than to maintain a certain [Hb]) should be determined in advance and assessment of success should be done at an interval (or intervals) appropriate to the underlying condition. In this setting, the physician and the patient must consider such questions as: What symptoms and signs are caused or aggravated by the anemia? Can these symptoms and signs be alleviated by red blood cell transfusions? What is the minimum level of hemoglobin at which the patient can function satisfactorily? Do the potential benefits of red blood cell transfusion outweigh the risks (and possibly the inconveniences) for this patient? In determining the risk-benefit ratio for a given patient, such factors as lifestyle, the presence of other medical disorders, the likely duration of the anemia and the patient's overall prognosis must be considered. For example, a patient may be willing to tolerate a very limited capacity for exertion if the anemia is likely to be temporary, but not if the anemia will be permanent.

In general, the risks per unit of red blood cell transfusion are the same in any setting. However, with the presence of severe chronic anemia, transfusion may lead to congestive heart failure, particularly in the elderly. In such cases, red blood cell transfusions must be administered very slowly and, in some patients, partial exchange transfusion may be performed. The administration of many red blood cell transfusions over a prolonged period will eventually lead to iron overload.

\subsection{Issues specific to red blood cell transfusion in children}

Principles guiding the decision to administer red blood cell transfusions to infants over 4 months of age and children are for the most part the same as for adults. Young children have lower [Hb] than adults - from $95-115 \mathrm{~g} / \mathrm{L}$ at 6 months of age to $115-125 \mathrm{~g} / \mathrm{L}$ at 2 years. These lower levels are thought to be due to the higher intra-erythrocyte levels of 2,3-DPG found in children.

The physiologic responses to anemia in childhood and the [Hb] at which transfusion becomes necessary have not been well studied. Two studies in Africa attempted to identify when transfusions should be given to prevent death in children with anemia and malaria. One involved 116 children under 5 years of age with mean hematocrit on admission of $0.14([\mathrm{Hb}]$ of $47 \mathrm{~g} / \mathrm{L})$. Children were randomly chosen to receive or not receive a transfusion of whole blood. There was no difference in hospital admissions and deaths in the "no transfusion" group versus the "transfusion" group. The second was a surveillance study in which data were collected over about 12 months on all children under 12 years of 
age ( $n=2433$ ) admitted to the pediatric ward of a Kenyan hospital. Transfusions (with whole blood) were administered according to routine practice and availability. Based on laboratory and clinical criteria, children with clinical signs of respiratory distress and $[\mathrm{Hb}]$ of less than $47 \mathrm{~g} / \mathrm{L}$ who were transfused had a lower fatality rate than those who were not. Among children without respiratory distress there was no association between receipt of blood transfusion and death, irrespective of [Hb] on admission. Although these studies are not definitive, they do support the clinical impression that asymptomatic children without underlying cardiorespiratory disease are able to tolerate very low [Hb] $(40-50 \mathrm{~g} / \mathrm{L})$ provided the anemia has developed slowly.

There are reports of successful outcomes of surgical procedures without the use of blood transfusion in the children of Jehovah's Witnesses. These procedures include open heart surgery in selected patients weighing less than $20 \mathrm{~kg}$.

For acute nonsurgical bleeding, the principles of resuscitation of a child are generally the same as for an adult. However, young infants are less able to tolerate rapid blood loss because of their limited ability to increase myocardial contractility in response to hypovolemia. Young infants rely primarily on increases in heart rate rather than contractility to increase CO. In addition, an apparently small amount of lost blood may be significant in a small child, because it may represent an important fraction of total blood volume $(80-90 \mathrm{~mL} / \mathrm{kg})$. Therefore, young infants, particularly those under 6 months of age, require earlier and more vigorous volume replacement and possibly earlier red blood cell replacement (e.g., after losses of $20-25 \%$ of total blood volume versus $30-35 \%$ in the older infant and child).

Pediatric patients are more likely than many adults to be long-term survivors; thus, in determining the balance between benefits and risks of transfusion, greater consideration must be given to long-term complications. For example, the transmission of viruses with long incubation periods (e.g., hepatitis $\mathrm{C}$ virus) and, for girls, the potential for hemolytic disease of the newborn in future pregnancies should they become alloimmunized to red blood cell antigens are considerations. Nevertheless, untreated chronic and severe anemia can lead to growth retardation in childhood.

\subsection{Recommendations regarding the transfusion of red blood cells}

5. A physician prescribing transfusion of red blood cells or plasma should be familiar with the indications for and the benefits and risk from the use of these fractions.

Level of evidence: N/A

6. Documentation that supports the administration of the red blood cells or plasma should be found in the patient's chart.

Level of evidence: N/A

7. Red blood cell transfusions should be administered primarily to prevent or alleviate symptoms, signs or morbidity due to inadequate tissue oxygen delivery (resulting from a low red blood cell mass).

Level of evidence: II

8. There is no single value of hemoglobin concentration that justifies or requires transfusion; an evaluation of the patient's clinical situation should also be a factor in the decision.

Level of evidence: II 
9. In the setting of acute blood loss, red blood cell transfusion should not be used to expand vascular volume when oxygen carrying capacity is adequate.

Level of evidence: II

10. Anemia should not be treated with red blood cell transfusions if alternative therapies with fewer potential risks are available and appropriate.

Level of evidence: II

\section{Autologous blood transfusion}

Autologous blood transfusion is a general term used to describe a procedure by which previously donated (or shed) blood is tranfused (or re-infused) into the same donor or patient. Autologous blood can be obtained by

- predonation (predeposit) of blood;

- perioperative normovolemic hemodilution (withdrawal of blood immediately before surgery, with volume replacement by crystalloid solutions) and subsequent reinfusion of the removed blood;

- intraoperative blood salvage;

- postoperative blood salvage.

The EWG considered only predonation.

A substantial proportion of patients who require blood are not candidates for autologous blood donation; for example, those with acute or chronic anemia; those with active infection; those requiring urgent surgery; small children; and some patients who require cancer surgery. Use of autologous blood reduces the likelihood that allogeneic blood will be used, and thus would be expected to reduce certain risks associated with blood transfusion - for example, the risk of exposure to transmissible infections such as HIV and hepatitis C; alloimmune hemolysis; allergic reactions; immunization to foreign antigens; graft-versus-host disease; and, possibly, immunosuppression. There is conflicting evidence as to whether predonation of autologous blood reduces postoperative infection because of avoidance of transfusion-associated immunosuppression. Also, autologous blood transfusion does not prevent other adverse effects: septicemia from bacterial contamination; non-hemolytic (febrile) transfusion reactions caused by plasma factors generated during blood storage; clerical or laboratory error leading to transfusion of the wrong blood unit; and circulatory overload. Because these risks remain, autologous blood should not be routinely reinfused to patients in the absence of an indication.

The risks associated with allogeneic blood that can be avoided by using autologous blood are rare. Furthermore, specific risks are associated with autologous blood. The risks from predeposited autologous blood include a higher likelihood of receiving blood in the postoperative period and adverse events associated with blood donation, particularly in cardiac patients. In addition, surgery may have to be rescheduled to avoid wastage of blood already collected.

There is insufficient evidence to indicate overall whether using autologous blood is more, equally or less safe than using allogeneic blood. Nevertheless, other considerations support the use of autologous blood for transfusion. First, it would theoretically prevent exposure to new infectious or other noxious agents that might contaminate the blood supply in the future. Second, there is a widespread perception among patients and health care workers that autologous blood is likely to be safer. Third, 
Table 2

Advantages and disadvantages of autologous blood transfusion

\begin{tabular}{|c|c|}
\hline Advantages & Disadvantages \\
\hline Reduces exposure to allogeneic blood & $\begin{array}{l}\text { Blood loss must be anticipated and a program established to } \\
\text { allow for blood predeposit or salvage }\end{array}$ \\
\hline $\begin{array}{l}\text { Reduces risk of } \\
\text { - transmissible viral infections } \\
\text { - alloimmune hemolysis } \\
\text { - allergic reactions } \\
\text { - immunization to foreign antigens } \\
\text { - graft-versus-host disease } \\
\text { - transfusion-induced immunosuppression }\end{array}$ & $\begin{array}{l}\text { Does not prevent adverse effects such as } \\
\text { - septicemia from bacterially contamined unit } \\
\text { - nonhemolytic transfusion reactions caused by plasma factors } \\
\text { generated during blood storage } \\
\text { - clerical or laboratory error leading to transfusion of the } \\
\text { wrong unit } \\
\text { - circulatory overload }\end{array}$ \\
\hline $\begin{array}{l}\text { Averts exposure to new or unknown infectious or other } \\
\text { noxious agents that might contaminate the blood } \\
\text { supply }\end{array}$ & $\begin{array}{l}\text { Increased likelihood of receiving blood in the postoperative } \\
\text { period } \\
\text { Cost is higher than with existing allogeneic program }\end{array}$ \\
\hline $\begin{array}{l}\text { Reduces demand for banked allogeneic blood, thus can } \\
\text { help alleviate blood shortages }\end{array}$ & Adds risk for adverse events associated with blood donation \\
\hline $\begin{array}{l}\text { Satisfies public demand and patients' desires that their } \\
\text { own blood be used }\end{array}$ & $\begin{array}{l}\text { Scheduling of surgery may have to be altered to permit au- } \\
\text { tologous blood donation or to avoid wasting blood already } \\
\text { collected }\end{array}$ \\
\hline
\end{tabular}

the interim report of the Commission of Inquiry on the Blood System in Canada has made several recommendations in favour of wider availability and use of autologous blood. Fourth, use of autologous blood reduces demand for banked allogeneic blood and thus helps alleviate blood shortages. (Note: At present, unused autologous blood units do not meet Canadian Red Cross donor criteria and, therefore, cannot be added to the general blood supply.) Fifth, autologous donation is helpful for elective surgical patients with rare blood groups or antibodies to multiple or high-incidence alloantigens.

The cost of providing autologous blood is higher than for providing allogeneic blood. Minimizing the waste of autologous units by focusing on patients. who are most likely to use blood is important in improving the cost-effectiveness of autologous blood.

A recent meta-analysis provides insight into the potential benefits and risks of a program for the predeposit of autologous blood (Table 2). This review of 6 randomized, controlled trials and 9 nonrandomized comparator-controlled studies revealed that the predeposit of autologous blood reduced the amount of allogeneic blood transfused (the odds ratio [OR] for the randomized, controlled trials was $0.17,95 \%$ confidence interval $[\mathrm{CI}] 0.08-0.32$ ). There was a direct relation between the proportion of patients transfused in the control group and the absolute benefit of predeposited autologous blood for reducing exposure to allogeneic blood (i.e., the benefit is greatest when the expected use of blood is highest). Interestingly, this meta-analysis also showed that patients. who predeposit autologous blood were significantly more likely to receive any blood product (including their own) than control patients (OR 6.69, 95\% CI 3.63-12.32). The authors offered 2 reasons for this observation: lower preoperative $[\mathrm{Hb}]$ and a more liberal transfusion policy for patients who had predeposited their own blood. This analysis suggests that patients who predeposit autologous blood may be at greater risk for certain complications associated with transfusion (e.g., septicemia from contaminated blood units, transfusion of the wrong blood unit due to laboratory or clerical error). 


\subsection{Recommendations regarding the use of autologous blood}

11. Predonation of autologous blood should be considered a therapeutic option for adolescents and adults undergoing elective surgery in which the likelihood of transfusion is substantial (i.e., $10 \%$ or more).

Level of evidence: III

12. Indications for the transfusion of autologous blood should be the same as those for allogeneic blood.

Level of evidence: III

\section{Plasma transfusion}

Plasma for transfusion is prepared by centrifuging anticoagulated whole blood from a single donor followed by storage at or below $-18^{\circ} \mathrm{C}$. Larger volumes of plasma may be collected using automated apheresis units and similarly stored. A typical unit of plasma has a volume of 200-250 mL if obtained from a whole blood donation or $400-600 \mathrm{~mL}$ when obtained by plasmapheresis. Plasma frozen within

Table 3

Alternatives to plasma

\begin{tabular}{ll}
\hline Type of product & Product \\
\hline Purified human plasma-derived concentrates* & Purified human albumin* \\
& Purified human immunoglobulin (IVIg) \\
& Purified human fibrinogen* \\
& Purified human factor VII* \\
& Purified human factor VIII:C* \\
& Purified human von Willebrand factor* \\
& Partly purified prothrombin complex (FEIBA) \\
& Partly purified human factor IX* \\
& Highly purified human factor IX* \\
& Purified human factor XI* \\
& Purified human factor XIII* \\
& Purified human protein C* \\
& Purified human antithrombin III* \\
& Purified human C1 esterase inhibitor* \\
& Fibrin glue (Tisseel) \\
& Purified porcine factor VIII:C \\
Purified animal plasma-derived concentrates* & Human factor VIII:C* \\
Recombinant proteins & Human factor VIIa \\
Synthetic replacement molecules & Amino acids (Aminosyn) \\
Pharmacologic agents & Pentastarch (Pentaspan) \\
& Desmopressin (DDAVP) \\
& Aminocaproic acid (Amicar) \\
& Tranexamic acid (Cyclokapron) \\
& Aprotinin (Trasylol) \\
\hline
\end{tabular}

*Available from the Canadian Red Cross Society according to a list distributed September 1996, subject to modifications as new products become available or existing products are deleted. 
$8 \mathrm{hrs}$ of donation contains at least $0.70 \mathrm{U} / \mathrm{mL}$ of factor VIII:C and is referred to as fresh-frozen plasma (FFP). In plasma frozen 8 to $72 \mathrm{hrs}$ after collection the concentration of coagulation factors $\mathrm{V}$ and VIII:C may be reduced as much as $15 \%$; it is usually referred to as frozen plasma (FP). In Canada, there is some regional variation as to which plasma products (FP and FFP) are available. However, in most therapeutic applications, there is little reason to choose one over the other, and the generic term plasma is used in this document. Cryosupernatant or cryo-poor plasma is the supernatant fluid obtained after preparation of a factor-VIII:C cryoprecipitate from plasma. It contains greatly reduced concentrations of factors V and VIII:C and other large-molecular-weight proteins such as fibrinogen, fibronectin and von Willebrand factor.

Excluding viruses such as human cytomegalovirus (CMV) and human T-cell lymphotropic virus type II (HTLV-II), which are transmitted only by infected leukocytes in cellular blood products, plasma carries the same risk of viral transmission per donor exposure as that of red blood cells. Virusinactivated purified or recombinant concentrates of many physiologically important plasma proteins are now commercially available. These products have been shown to be safer and more effective than plasma in correcting specific protein or coagulation factor deficiencies. Hence, the consensus in published guidelines is that plasma transfusion should be avoided when a safer and more effective product can be used to achieve the same therapeutic goal (Table 3). This applies specifically to:

- intravascular volume expansion or repletion, where crystalloids, synthetic colloids or purified human albumin solutions are preferred (including therapeutic plasmapheresis where plasma should not be used as routine replacement fluid);

- the correction of hypoalbuminemia or protein malnutrition, where purified human albumin or synthetic amino acid solutions are preferred;

- the correction of hypogammaglobulinemia, where purified human immunoglobulin concentrates are preferred;

- the treatment of hemophilia and von Willebrand's disease, where desmopressin (DDAVP) or existing virus-free factor concentrates are preferred;

- the treatment of any other isolated congenital procoagulant or anticoagulant factor deficiency, where virus-inactivated or recombinant factor concentrates are preferred if they exist. 